\title{
VARIABLE SELECTION FOR CENSORED QUANTILE REGRESION
}

\author{
Huixia Judy Wang ${ }^{1}$, Jianhui Zhou ${ }^{2}$ and $\mathrm{Yi} \mathrm{Li}^{3}$ \\ North Carolina State University ${ }^{1}$, University of Virginia ${ }^{2}$ and Harvard University ${ }^{4}$
}

Abstract: Quantile regression has emerged as a powerful tool in survival analysis as it directly links the quantiles of patients' survival times to their demographic and genomic profiles, facilitating the identification of important prognostic factors. In view of limited work on variable selection in the context, we develop a new adaptive-lasso-based variable selection procedure for quantile regression with censored outcomes. To account for random censoring for data with multivariate covariates, we employ the ideas of redistribution-of-mass and effective dimension reduction. Asymptotically our procedure enjoys the model selection consistency, that is, identifying the true model with probability tending to one. Moreover, as opposed to the existing methods, our new proposal requires much fewer stringent assumptions, leading to more accurate variable selection even with small or moderate sample sizes. The analysis of a real cancer clinical trial demonstrates that our procedure can identify and distinguish important factors associated with patient subpopulations characterized by short or long survivals, which is of particular interest to oncologists.

Key words and phrases: Conditional Kaplan-Meier, dimension reduction, kernel, quantile regression, survival analysis, variable selection. 